UND-HEP-00-BIG 03

hep-ph/0005278

\title{
Analytical Heavy Quark Expansion in the 't Hooft Model
}

\author{
Matthias Burkardt $^{a}$ and Nikolai Uraltsev ${ }^{b, c}$ \\ ${ }^{a}$ Dept. of Physics, New Mexico State Univ., Las Cruces, NM 88003-0001, U.S.A. \\ ${ }^{b}$ Physics Dept., Univ. of Notre Dame du Lac, Notre Dame, IN 46556, U.S.A. \\ ${ }^{c}$ Petersburg Nuclear Physics Inst., Gatchina, St. Petersburg, 188350, Russia
}

\begin{abstract}
We present a number of exact relations for the heavy quark limit and develop an analytical $1 / m_{Q}$ expansion for heavy mesons in the 't Hooft model. Among the new results are relation $3 \mu_{\pi}^{2}=\bar{\Lambda}^{2}-m_{\mathrm{sp}}^{2}+\beta^{2}, 1 / m_{Q}$ corrections to the decay constants, to the kinetic expectation values and $1 / m_{Q}^{2}$ nonperturbative corrections to the $B \rightarrow D$ amplitude at zero recoil. The properties of the IW functions are addressed and the small velocity sum rules are verified.
\end{abstract}




\section{Introduction}

Heavy quark symmetry and the heavy quark expansion have played an important role in understanding weak decays of charm and beauty hadrons allowing extraction of the fundamental parameters of the Standard Model. Beauty and, in particular, charm quarks are not infinitely heavy even in the crude approximation. In practice, $1 / m_{Q}$ corrections to the strict $m_{Q} \rightarrow \infty$ limit often constitute the main limitation. Even in a few cases where the first terms are known, a question remains about the convergence of the employed $1 / m_{Q}$ expansion. (For a review and further references, see Ref. [1].)

In such a situation it is advantageous to have a model laboratory where both $1 / m_{Q}$ corrections, and the whole finite- $m_{Q}$ amplitudes can be evaluated exactly. On the one hand, this allows to trace in detail how the methods used for actual QCD work in a simplified setting. On the other hand, it has been empirically observed that certain quantities in the heavy flavor hadrons suffer from numerically large power corrections, whilst others seem robust against finite mass effects. Studying this in the toy models can help gaining some insights which can be applied in various studies of actual QCD, including those based on the QCD sum rules technique or lattice computations.

One such solvable model, which has been applied to a variety of strong interaction phenomena in the past is the 't Hooft model, QCD in $1+1$ dimensions at large number of colors $N_{c} \rightarrow \infty$ [2]. The 't Hooft model has two important features that resemble real $\mathrm{QCD}_{3+1}$ phenomenologically: confinement and spontaneous chiral symmetry breaking. Since the underlying microscopic mechanisms for these effects in this model are quite different from the respective mechanisms in $\mathrm{QCD}_{3+1}$, it is probable that $\mathrm{QCD}_{1+1}$ is of little help to understand the origin of these features in real QCD, but it may nevertheless be very valuable to better understand the consequences for other observables.

In this paper we will focus on the $m_{Q} \rightarrow \infty$ limit of the 't Hooft model. Even though we were not able to solve this limit analytically, we succeeded to derive a number of exact relations involving terms that appear in the $1 / m_{Q}$ expansion. In Section 2 we will discuss some general features of this model. In Section 3 the static limit $m_{Q} \rightarrow \infty$ will be explored and we will derive a number of relations involving 't Hooft wavefunctions in this limit. These relations will be applied in Section 4 to derive some exact results for power corrections in this model. Section 5 gives the conclusions.

\section{The 't Hooft Model}

$\mathrm{QCD}_{1+1}$ is based on the Lagrangian

$$
\mathcal{L}=\sum_{q} \bar{q}\left(i \gamma^{\mu} D_{\mu}-m_{q}\right) q-\frac{1}{2 g_{s}^{2}} \operatorname{Tr} G^{\mu \nu} G_{\mu \nu}
$$


defined in one space and one time dimension, i.e. $\mu, \nu=0,1$ and all fields depend only on two space-time coordinates. In the light-cone gauge, $A^{+}=0$, as in any other axial gauge, the non-Abelian term in the field strength tensor $G_{\mu \nu}=i\left[D_{\mu}, D_{\nu}\right]=$ $\partial_{\mu} A_{\nu}-\partial_{\nu} A_{\mu}+i\left[A_{\mu}, A_{\nu}\right]$ vanishes, which drastically simplifies the dynamics of the model. This allows one to eliminate the only non-vanishing component of the gauge field $A^{-}$, by means of the Poisson equation

$$
-\partial_{-}^{2} A_{a}^{-}=g_{s}^{2} J_{a}^{+},
$$

where we have introduced light-cone coordinates $x^{ \pm}=\frac{1}{\sqrt{2}}\left(x^{0} \pm x^{1}\right)$. In two dimensions there are no dynamical gluons and, after solving the Poisson equation (2), the only remnant of the gluon field is a Coulomb like instantaneous interaction among the quarks. Of course, in one space dimension, the 'Coulomb' interaction is linearly confining. It is this feature which makes $\mathrm{QCD}_{1+1}$ so attractive if one is interested in studying models which exhibit infrared slavery.

An additional simplification occurs in the large $N_{c}$ limit

$$
N_{c} \rightarrow \infty \quad \beta^{2} \equiv \frac{g_{s}^{2} N_{c}}{2 \pi} \quad \text { fixed }
$$

where sea quarks (more precisely, quark loops) are suppressed and only planar diagrams survive. Therefore, in a light-cone Fock space expansion, the valence $\bar{q} q$ approximation for mesons becomes exact, and the two body equation for the quark distribution amplitude $\varphi_{n}(x)$ of mesons (the 't Hooft equation [2]) reads

$$
\begin{aligned}
M_{n}^{2} \varphi_{n}(x) & =\left[\frac{m_{1}^{2}-\beta^{2}}{x}+\frac{m_{2}^{2}-\beta^{2}}{1-x}\right] \varphi_{n}(x)-\beta^{2} \int_{0}^{1} \mathrm{~d} y \frac{\varphi_{n}(y)}{(y-x)^{2}} \\
& \equiv\left[\frac{m_{1}^{2}-\beta^{2}}{x}+\frac{m_{2}^{2}-\beta^{2}}{1-x}\right] \varphi_{n}(x)+V \varphi_{n}(x)
\end{aligned}
$$

Here $x$ denotes the light-cone momentum fraction carried by the quark and $M_{n}$ is the invariant mass of the meson.

Eq. (4) has a very physical interpretation: the light-cone energy of the $q \bar{q}$ pair consists of a sum of the light-cone kinetic energies of the quark and antiquark plus an interaction term $V$. The integral operator $V$ can be interpreted as the momentum space representation of a linear potential. The singularity of the QCDCoulomb interaction in Eq. (田) is regularized using the principal value prescription, with $\int_{0}^{1} d y \frac{1}{(x-y)^{2}}=-\left(\frac{1}{x}+\frac{1}{1-x}\right)$.

For practical purposes, it is convenient to rewrite Eq. (4) into the form where the singularity of the interaction term is less severe:

$$
M_{n}^{2} \varphi_{n}(x)=\left(\frac{m_{1}^{2}}{x}+\frac{m_{2}^{2}}{1-x}\right) \varphi_{n}(x)-\beta^{2} \int_{0}^{1} \mathrm{~d} y \frac{\varphi_{n}(y)-\varphi_{n}(x)}{(y-x)^{2}} .
$$


Note that Eq. (5) can also be obtained as a variational equation for the functional (Hamiltonian) defined by the quadratic form

$$
\langle n|\mathcal{H}| n\rangle=\int_{0}^{1} \mathrm{~d} x\left(\frac{m_{1}^{2}}{x}+\frac{m_{2}^{2}}{1-x}\right) \varphi_{n}^{2}(x)+\frac{\beta^{2}}{2} \int_{0}^{1} \mathrm{~d} x \mathrm{~d} y \frac{\left(\varphi_{n}(y)-\varphi_{n}(x)\right)^{2}}{(y-x)^{2}} .
$$

The interaction term in Eq. (6) is non-negative and the kinetic term is minimized for $\frac{x}{(1-x)}=\frac{m_{1}}{m_{2}}$, i.e. when the ratio of momentum fractions carried by the quark and antiquark equals their ratio of bare masses. Therefore the variational formulation Eq. (6) of the 't Hooft equation yields a lower bound

$$
M_{n}>m_{1}+m_{2}
$$

(the inequality is saturated only for the massless pion where $m_{1}=m_{2}=0$ and at $n=0$, with $\varphi_{\pi}(x)=1$ ). The mass of a bound state exceeds the sum of the bare masses of the constituents, the fact expected semiclassically.

A certain parity relation proves to be useful, it was first given in Ref. [3] and reportedly ascends to 't Hooft. We define operator $K$ as

$$
K \varphi(x)=\int_{0}^{1} \mathrm{~d} y \frac{\varphi(y)}{y-x} .
$$

Then the following commutation relation holds:

$$
[\mathcal{H}, K]=\frac{m_{1}^{2}}{x} \int_{0}^{1} \frac{\mathrm{d} y}{y} \varphi(y)-\frac{m_{2}^{2}}{1-x} \int_{0}^{1} \frac{\mathrm{d} y}{1-y} \varphi(y) .
$$

Since for eigenstates the expectation value of the commutator of any operator $A$ with Hamiltonian vanishes,

$$
\langle n|[\mathcal{H}, A]| n\rangle=0
$$

one has

$$
0=\langle n|[\mathcal{H}, K]| n\rangle=m_{1}^{2}\left(\int_{0}^{1} \frac{\mathrm{d} y}{y} \varphi_{n}(y)\right)^{2}-m_{2}^{2}\left(\int_{0}^{1} \frac{\mathrm{d} y}{1-y} \varphi_{n}(y)\right)^{2} .
$$

Therefore,

$$
m_{2} \int_{0}^{1} \frac{\mathrm{d} y}{1-y} \varphi_{n}(y)=-P_{n} m_{1} \int_{0}^{1} \frac{\mathrm{d} y}{y} \varphi_{n}(y),
$$

and $P_{n}$ is identified with the parity of the eigenstate $n$. This identification is confirmed by comparing with explicit expressions for the matrix elements of the pseudoscalar and scalar densities between the state $n$ and the vacuum [3]

$$
\begin{aligned}
\left\langle 0\left|\bar{q}_{2} q_{1}\right| n\right\rangle & =\sqrt{\frac{N_{c}}{4 \pi}} \int_{0}^{1} d x\left(\frac{m_{1}}{x}-\frac{m_{2}}{1-x}\right) \varphi_{n}(x) \\
\left\langle 0\left|\bar{q}_{2} i \gamma_{5} q_{1}\right| n\right\rangle & =\sqrt{\frac{N_{c}}{4 \pi}} \int_{0}^{1} d x\left(\frac{m_{1}}{x}+\frac{m_{2}}{1-x}\right) \varphi_{n}(x) .
\end{aligned}
$$


Using Eq. (12), it is easy to see that $\left\langle 0\left|\bar{q}_{2} q_{1}\right| n\right\rangle \neq 0$ only for states with $P_{n}=1$ and $\left\langle 0\left|\bar{q}_{2} i \gamma_{5} q_{1}\right| n\right\rangle \neq 0$ only for states with $P_{n}=-1$.

Integrating the 't Hooft equation over $x$ and using the parity relation (12) we get

$$
\begin{aligned}
\int_{0}^{1} \frac{\mathrm{d} x}{x} \varphi_{n}(x) & =\frac{M_{n}^{2}}{m_{1}\left(m_{1}-P_{n} m_{2}\right)} \int_{0}^{1} \mathrm{~d} x \varphi_{n}(x), \\
\int_{0}^{1} \frac{\mathrm{d} x}{1-x} \varphi_{n}(x) & =\frac{M_{n}^{2}}{m_{2}\left(m_{2}-P_{n} m_{1}\right)} \int_{0}^{1} \mathrm{~d} x \varphi_{n}(x) .
\end{aligned}
$$

Similarly, integrating the 't Hooft equation multiplied by $x$ yields

$$
\begin{aligned}
\int_{0}^{1} \mathrm{~d} x x \varphi_{n}(x) & =\left[\frac{m_{1}^{2}-m_{2}^{2}}{M_{n}^{2}}+\frac{m_{2}}{m_{2}-P_{n} m_{1}}\right] \int_{0}^{1} \mathrm{~d} x \varphi_{n}(x)-\frac{\beta^{2}}{M_{n}^{2}} \int_{0}^{1} \mathrm{~d} x \ln \frac{1-x}{x} \varphi_{n}(x), \\
\int_{0}^{1} \mathrm{~d} x(1-x) \varphi_{n}(x) & =\left[\frac{m_{2}^{2}-m_{1}^{2}}{M_{n}^{2}}+\frac{m_{1}}{m_{1}-P_{n} m_{2}}\right] \int_{0}^{1} \mathrm{~d} x \varphi_{n}(x)+\frac{\beta^{2}}{M_{n}^{2}} \int_{0}^{1} \mathrm{~d} x \ln \frac{1-x}{x} \varphi_{n}(x) .
\end{aligned}
$$

Another useful relation for the 't Hooft equation which holds for arbitrary quark masses was derived by Burkardt [4] and is often referred to as the virial theorem. Let us denote

$$
D=x \frac{\mathrm{d}}{\mathrm{dx}}, \quad \tilde{D}=(1-x) \frac{\mathrm{d}}{\mathrm{d}(1-\mathrm{x})} .
$$

A direct computation yields for the commutator of $D$ with the interaction operator $V$ in the 't Hooft equation the following result:

$$
[D, V]=-V
$$

and, therefore,

$$
[D, \mathcal{H}]=-\mathcal{H}+\frac{m_{2}^{2}-\beta^{2}}{(1-x)^{2}}
$$

Likewise

$$
[\tilde{D}, \mathcal{H}]=-\mathcal{H}+\frac{m_{1}^{2}-\beta^{2}}{x^{2}} .
$$

Using again the fact that the expectation value of the commutator of any operator with Hamiltonian vanishes in an energy eigenstate, these two commutation relations yield the relation which holds for any eigenfunction:

$$
M_{n}^{2}=\left(m_{1}^{2}-\beta^{2}\right)\left\langle\frac{1}{x^{2}}\right\rangle=\left(m_{2}^{2}-\beta^{2}\right)\left\langle\frac{1}{(1-x)^{2}}\right\rangle .
$$

The 't Hooft equations describing mesons with one heavy quark $Q$ acquire new symmetry which entails one to a number of additional relations. In the rest of the paper we study these relations in the static limit $\left(m_{Q} \rightarrow \infty\right)$ and develop the $1 / m_{Q}$ expansion around this limit.

\footnotetext{
${ }^{1}$ As it stands, Eq. (19) is valid only for $m^{2}>\beta^{2}$. For $m^{2} \leq \beta^{2}$ it can be replaced by a subtracted version [4].
} 


\section{Static version of the 't Hooft equation}

In the heavy quark limit, i.e. when the mass of the quark $m_{1} \rightarrow \infty$, the meson wave functions become sharply peaked near $x \rightarrow 1$, since most of the momentum is then carried by this quark. Therefore, in order to study the $m_{1} \equiv m_{Q} \rightarrow \infty$ limit, it is convenient to introduce the nonrelativistic variables $M_{n}=m_{Q}+\tilde{\epsilon}_{n}, t=(1-x) m_{Q}$ and $\Psi_{n}(t)=\frac{1}{\sqrt{m_{Q}}} \varphi_{n}\left(1-\frac{t}{m_{Q}}\right)$. In these variables the 't Hooft equation (4) takes the form

$$
\left(\tilde{\epsilon}_{n}+\frac{\tilde{\epsilon}_{n}^{2}+\beta^{2}}{2 m_{Q}}\right) \Psi_{n}(t)=\left(\frac{m_{\mathrm{sp}}^{2}-\beta^{2}}{2 t}+\frac{t}{2} \frac{1-\frac{\beta^{2}}{m_{Q}^{2}}}{1-\frac{t}{m_{Q}}}\right) \Psi_{n}(t)-\frac{\beta^{2}}{2} \int_{0}^{m_{Q}} \mathrm{~d} s \frac{\Psi_{n}(s)}{(t-s)^{2}} .
$$

We assume that in the notations of the previous section $m_{1}=m_{Q}$ is large, and $m_{\mathrm{sp}}=m_{2}$ will be denoted simply as $m$ below. The limit $m_{Q} \gg \beta$ is obtained by expanding the second term in the r.h.s. of Eq. (20) in $t / m_{Q}$ and extending it to the interval $[0, \infty): 2$

$$
\begin{gathered}
\left(\tilde{\epsilon}_{n}+\frac{\tilde{\epsilon}_{n}^{2}+\beta^{2}}{2 m_{Q}}\right) \Psi_{n}(t)=\left(\frac{m^{2}-\beta^{2}}{2 t}\right) \Psi_{n}(t)+\left(1-\frac{\beta^{2}}{m_{Q}^{2}}\right)\left[\frac{t}{2}+\frac{t^{2}}{2 m_{Q}}+\frac{t^{3}}{2 m_{Q}^{2}}+\ldots\right] \Psi_{n}(t) \\
-\frac{\beta^{2}}{2} \int_{0}^{\infty} \mathrm{d} s \frac{\Psi_{n}(s)}{(t-s)^{2}} .
\end{gathered}
$$

Performing $1 / m_{Q}$ expansion, it is convenient to study the eigenvalues

$$
\epsilon_{n} \equiv \tilde{\epsilon}_{n}+\frac{\tilde{\epsilon}_{n}^{2}+\beta^{2}}{2 m_{Q}}
$$

of the equation themselves rather than directly $\tilde{\epsilon}_{n}$. This will be assumed later when we study $1 / m_{Q}$ corrections to the static limit. (The explicit factor $1-\beta^{2} / m_{Q}^{2}$ can be eliminated by properly rescaling $t$.)

The static limit is obtained neglecting all terms suppressed by inverse powers of $m_{Q}$ [5, 6, 迆:

$$
\epsilon_{n} \Psi_{n}(t)=\frac{m^{2}-\beta^{2}}{2 t} \Psi_{n}(t)+\frac{t}{2} \Psi_{n}(t)-\frac{\beta^{2}}{2} \int_{0}^{\infty} \mathrm{d} s \frac{\Psi_{n}(s)}{(t-s)^{2}} .
$$

This is a stationary Schrödinger-type equation for the one-dimensional system with the static Hamiltonian

$$
\mathcal{H}=\mathcal{H}_{\text {stat }}=\frac{m^{2}-\beta^{2}}{2 t}+\frac{t}{2}-\frac{\beta^{2}}{2} \int_{0}^{\infty} \frac{\mathrm{d} s}{(t-s)^{2}} .
$$

Similar to Eq. (6), the expectation value of the Hamiltonian over a state $\Psi_{n}$ can be written in the form

$$
\left\langle n\left|\mathcal{H}_{\text {stat }}\right| n\right\rangle=\int_{0}^{\infty} \mathrm{d} t\left(\frac{m^{2}}{2 t}+\frac{t}{2}\right) \Psi_{n}^{2}(t)+\frac{\beta^{2}}{4} \int_{0}^{\infty} \mathrm{d} s \mathrm{~d} t \frac{\left(\Psi_{n}(s)-\Psi_{n}(t)\right)^{2}}{(s-t)^{2}} .
$$

\footnotetext{
${ }^{2}$ Extending the interval to $[0, \infty)$ introduces errors that are only $\mathcal{O}\left(1 / m_{Q}^{4}\right)$.
} 
Since $\frac{m^{2}}{2 t}+\frac{t}{2} \geq m$, Eq. (25) suggests that $\epsilon_{n}>m$. This lower bound follows also directly from the general lower bound Eq. (17).

The large- $t$ asymptotics of the static eigenfunctions is obtained directly:

$$
\Psi_{n}(t) \stackrel{t \rightarrow \infty}{\longrightarrow} \frac{\beta^{2} F^{(n)}}{t^{3}}
$$

where

$$
F^{(n)} \equiv \int_{0}^{\infty} \mathrm{d} t \Psi_{n}(t)=\lim _{m_{Q} \rightarrow \infty} \sqrt{\frac{\pi m_{Q}}{N_{c}}} f_{n},
$$

and $f_{n}$ is the usual annihilation constant of a meson [3]. The combination $F^{(n)}$ has finite large- $N_{c}$ and large- $m_{Q}$ limits. Together with various moments (integrals of $\Psi_{n}^{2}(t)$ with powers of $t$ ) it plays an important role in the heavy quark expansion. For future use, it is convenient to define a set of operators acting on $\Psi(t)$,

$$
J_{l} \Psi=\int_{0}^{\infty} \mathrm{d} t t^{l} \Psi(t), \quad J_{0} \equiv J
$$

and matrix elements

$$
F_{l}^{(n)}=\int_{0}^{\infty} \mathrm{d} t t^{l} \Psi_{n}(t)
$$

Then, for example, $\left(F^{(n)}\right)^{2}=\left\langle n\left|J_{0}\right| n\right\rangle$. Of course, the integrals in Eqs. (28) and (29) literally converge only for $-1 \leq l<2$.

The static analogue of the operator $K$ in Eq. (8) takes the form

$$
K \Psi(t)=\int_{0}^{\infty} \mathrm{d} s \frac{\Psi(s)}{s-t}
$$

and its commutator with the Hamiltonian

$$
[H, K]=\frac{m^{2}}{2 t} \int_{0}^{\infty} \frac{\mathrm{d} s}{s}-\frac{1}{2} \int_{0}^{\infty} \mathrm{d} s=\frac{m^{2}}{2 t} J_{-1}-\frac{1}{2} J_{0} .
$$

For any energy eigenstate $n$, the equation $\langle n|[H, K]| n\rangle=0$ holds, and we thus find

$$
m^{2}\left|\int_{0}^{\infty} \frac{\mathrm{d} t}{t} \Psi_{n}(t)\right|^{2}=\left|\int_{0}^{\infty} \mathrm{d} t \Psi_{n}(t)\right|^{2}
$$

or

$$
m \int_{0}^{\infty} \frac{\mathrm{d} t}{t} \Psi_{n}(t)=-P_{n} \int_{0}^{\infty} \mathrm{d} t \Psi_{n}(t)
$$

i.e. $m F_{-1}^{(n)}=-P_{n} F^{(n)}$. Hereafter we call states for which $P_{n}=-1$ and $P_{n}=1 \quad P$ odd and $P$-even, respectively. Of course, Eq. (33) can also be obtained directly by applying the heavy quark limit to the finite mass parity relation Eq. (12), which is the reason why corrections to the static limit do not spoil the parity classification. 
Another relation among the moments of the wavefunction can be obtained by integrating the static equation (23) from 0 to $\infty$, yielding

$$
2 \epsilon_{n} F^{(n)}=m^{2} F_{-1}^{(n)}+F_{1}^{(n)}
$$

which with the help of the above parity relation can be written as

$$
F_{1}^{(n)}=\left(2 \epsilon_{n}+m P_{n}\right) F^{(n)} .
$$

Non-diagonal matrix elements of $K$ are also expressed in terms of $\epsilon$ and $F$ :

$$
\langle n|K| l\rangle=\frac{P_{n} P_{l}-1}{2} \frac{1}{\epsilon_{n}-\epsilon_{l}} F^{(n)} F^{(l)} .
$$

Certain useful relations emerge also from the commutation relation

$$
[\mathcal{H}, t]=-\frac{\beta^{2} K}{2},
$$

and the obvious commutator $[t, K]=-J_{0}$. Thus, for example, $[t,[t, \mathcal{H}]]=-\frac{\beta^{2}}{2} J_{0}$ and hence $\langle n|[t,[t, \mathcal{H}]]| n\rangle=-\frac{\beta^{2}}{2}\left(F^{(n)}\right)^{2}$. 自

In the following, we will derive a tower of relations among the moments of $\Psi_{n}^{2}$. For this purpose, we consider the operators

$$
D_{n}=t^{n} \frac{\mathrm{d}}{\mathrm{d} t}, \quad D_{1}=t \frac{\mathrm{d}}{\mathrm{d} t} \equiv D .
$$

A direct computation yields

$$
\left[D_{n}, \mathcal{H}\right]=-n t^{n-1} \mathcal{H}+\frac{n-1}{2}\left(m^{2}-\beta^{2}\right) t^{n-2}+\frac{n+1}{2} t^{n}-\frac{\beta^{2}}{2} \sum_{k=0}^{n-3}(k+1)(n-k-2) t^{n-k-3} J_{k} .
$$

For $n=0,1,2$ the last sum is absent. The first few relations take the form

$$
\begin{array}{rlrl}
{\left[\frac{\mathrm{d}}{\mathrm{d} t}, \mathcal{H}\right.} & =-\frac{m^{2}-\beta^{2}}{2 t^{2}}+\frac{1}{2} & & n=0 \\
{\left[t \frac{\mathrm{d}}{\mathrm{d} t}, \mathcal{H}\right]} & =-\mathcal{H}+t & & n=1 \\
{\left[t^{2} \frac{\mathrm{d}}{\mathrm{d} t}, \mathcal{H}\right]} & =-2 t \mathcal{H}+\frac{1}{2}\left(m^{2}-\beta^{2}\right)+\frac{3}{2} t^{2} & n & =2 \\
{\left[t^{3} \frac{\mathrm{d}}{\mathrm{d} t}, \mathcal{H}\right.} & =-3 t^{2} \mathcal{H}+\left(m^{2}-\beta^{2}\right) t+2 t^{3}-\frac{\beta^{2}}{2} J_{0} & n \\
{\left[t^{4} \frac{\mathrm{d}}{\mathrm{d} t}, \mathcal{H}\right]} & =-4 t^{3} \mathcal{H}+\frac{3}{2}\left(m^{2}-\beta^{2}\right) t^{2}+\frac{5}{2} t^{4}-\beta^{2} t J_{0}-\beta^{2} J_{1} & n & =4 .
\end{array}
$$

Taking the expectation values of the operator relations in Eqs. (39) we obtain the moments $\left\langle t^{n}\right\rangle$ in terms of the bound-state energies and decay constants $F$ :

$$
\begin{array}{rlrl}
n=1 & \langle t\rangle & =\epsilon \\
n=2 & 3\left\langle t^{2}\right\rangle & =4\langle t\rangle^{2}-\left(m^{2}-\beta^{2}\right) \\
n=3 & 4\left\langle t^{3}\right\rangle & =6\left\langle t^{2}\right\rangle\langle t\rangle-2\left(m^{2}-\beta^{2}\right)\langle t\rangle+\beta^{2} F_{0}^{2} \\
n=4 & 5\left\langle t^{4}\right\rangle & =8\left\langle t^{3}\right\rangle\langle t\rangle-3\left(m^{2}-\beta^{2}\right)\left\langle t^{2}\right\rangle+4 \beta^{2} F_{0} F_{1} .
\end{array}
$$

\footnotetext{
${ }^{3}$ This relation is the so-called fourth sum rule for the Darwin operator.
} 
Note that $n \geq 5$ does not literally lead to meaningful relations since they would involve divergent terms. The case $n=0$ yields the relation

$$
\left(m^{2}-\beta^{2}\right) \int_{0}^{\infty} \frac{\mathrm{d} t}{t^{2}} \Psi^{2}(t)=1
$$

i.e. the virial theorem Eq. (19) for the light quark in the limit where the other quark is static. As we have mentioned above, it is literally valid at $m>\beta$, for smaller $m$ it can be understood, for example, as an analytic continuation in the mass of light antiquark or in a subtracted form. As we will show later, these moments are important in the $1 / m_{Q}$ expansion since the leading $1 / m_{Q}$ corrections in Eq. (21) are simple powers of $t$.

The relation $\langle t\rangle=\epsilon_{n}$ is also a direct consequence of the virial equation (19) (the one which involves $m_{Q}^{2}$ ) expanded to the first nontrivial order in $1 / m_{Q}$. It was first derived in Ref. [6] (see also [0]). In QCD the bound-state energies $\epsilon_{n}$ are usually denoted by $\bar{\Lambda}_{n}$.

The next important parameter of the heavy quark expansion is the kinetic expectation value $\mu_{\pi}^{2}=\frac{1}{2 M_{H_{Q}}}\left\langle H_{Q}\left|\bar{Q}(i \vec{D})^{2} Q\right| H_{Q}\right\rangle$. In the limit $m_{Q} \rightarrow \infty$ the operator of the spacelike momentum takes the simple form, since the $Z$-graph contributions can be neglected, the light-cone combination of momentum is given by $x M_{H_{Q}}$ and the time component can be excluded using the equation of motion $D_{0} Q=m_{Q} Q$. In the 't Hooft model, in the limit $m_{Q} \rightarrow \infty$ this immediately leads to

$$
\mu_{\pi}^{2}=\int_{0}^{\infty} \mathrm{d} t(t-\bar{t})^{2} \Psi^{2}(t)=\left\langle t^{2}\right\rangle-\langle t\rangle^{2} .
$$

Combining the $n=2$ relation in Eq. (40) with the virial equation $(n=1)$ one finds

$$
3 \mu_{\pi}^{2}=\bar{\Lambda}^{2}-m^{2}+\beta^{2}, \quad \bar{\Lambda} \equiv \epsilon_{n},
$$

for any bound state.

In the absence of actual chromomagnetic field in $D=2$ the next operator is represented by the Darwin term

$$
\rho_{D}^{3}=\frac{1}{2 M_{H_{Q}}}\left\langle H_{Q}\left|\bar{Q}\left(-\frac{1}{2} \vec{D} \vec{E}\right) Q\right| H_{Q}\right\rangle=\frac{\beta^{2} F^{2}}{4}
$$

(the last relation is obtained using the equation of motion for the gauge field, and factorization valid at $\left.N_{c} \rightarrow \infty\right)$. At the same order a nonlocal zero-momentum correlator of the kinetic operators appears as well, which will be addressed in the next section.

Before proceeding to the IW functions, let us mention an upper bound on the decay constants $F^{(n)}$. It is obtained using one of the Sobolev's inequalities bounding the $L_{\infty}$ norm via $L_{2}$ and $L_{2}^{1}$ norms in one dimension:

$$
|f(a)| \leq 2^{1 / 2}\left[\int \mathrm{d} z|f(z)|^{2}\right]^{1 / 4}\left[\int \mathrm{d} z\left|f^{\prime}(z)\right|^{2}\right]^{1 / 4} \quad \text { for any } a .
$$


In terms of the Fourier transform of $f(z), \Psi(t)$ it takes the form

$$
\left|\int \mathrm{d} t \Psi(t)\right| \leq \pi^{1 / 2}\left[\int \mathrm{d} t \Psi^{2}(t)\right]^{1 / 4}\left[\int \mathrm{d} t t^{2} \Psi^{2}(t)\right]^{1 / 4} .
$$

Applied to the static wavefunction it reads

$$
F \leq \sqrt{\pi}\left(\bar{\Lambda}^{2}+\mu_{\pi}^{2}\right)^{1 / 4}=\sqrt{\pi}\left(\frac{4 \bar{\Lambda}^{2}-m^{2}+\beta^{2}}{3}\right)^{1 / 4}
$$

or

$$
\rho_{D}^{3}<\frac{\pi}{4} \beta^{2}\left(\bar{\Lambda}^{2}+\mu_{\pi}^{2}\right)^{1 / 2}
$$

These bounds are a $D=2$ counterpart of the bounds discussed in QCD in Ref. [8].

Since the inequality in Eq. (46) is saturated only by functions of the form $1 /\left(c+t^{2}\right)$, a solution of the 't Hooft equation cannot saturate the bound (47). It is interesting, however, that for the ground states with light spectator quarks, $m \lesssim \beta$ the decay constant $F$ numerically almost saturates the bound, within only a few percent.

The operator $t$ plays a special role for the static equation (23): the first and the last terms in $\mathcal{H}_{\text {stat }}$ are homogeneous functionals of rank -1 with respect to $t$, while the term $\propto t / 2$ has rank +1 . It breaks the dilatation invariance of the eigenstate problem. This operator is the analogue of the operator $\mathcal{D}$ of Ref. [9] representing the part of the full trace of the energy-momentum tensor $\theta_{\mu \mu}$ associated with the light degrees of freedom (Sect. II, Eq. (9)). Likewise, there are many relations for various observables in the 't Hooft model, involving the operator $t$. Here we consider matrix elements of $t$.

Using the commutator Eq. (36) we write

$$
\begin{aligned}
& \langle k|t| n\rangle=\frac{1}{\epsilon_{k}-\epsilon_{n}}\langle k|[\mathcal{H}, t]| n\rangle=-\frac{\beta^{2}}{2\left(\epsilon_{k}-\epsilon_{n}\right)}\langle k|K| n\rangle=-\frac{\beta^{2}}{2\left(\epsilon_{k}-\epsilon_{n}\right)^{2}}\langle k|[\mathcal{H}, K]| n\rangle= \\
& =-\frac{\beta^{2}}{4\left(\epsilon_{k}-\epsilon_{n}\right)^{2}}\left(m^{2} F_{-1}^{(k)} F_{-1}^{(n)}-F_{0}^{(k)} F_{0}^{(n)}\right)=\frac{\beta^{2}}{4\left(\epsilon_{k}-\epsilon_{n}\right)^{2}} F_{0}^{(k)} F_{0}^{(n)}\left(1-P_{k} P_{n}\right),
\end{aligned}
$$

where relations (31) and (33) have also been used. This can be cast into the form

$$
\langle k|t-\mathcal{H}| n\rangle=\frac{\beta^{2}}{2\left(\epsilon_{k}-\epsilon_{n}\right)^{2}} F_{0}^{(k)} F_{0}^{(n)}\left(\frac{1-(-1)^{k-n}}{2}\right)
$$

which embeds both $k=n$ and $k \neq n$ (we have used the fact that $P_{k} P_{n}=(-1)^{k-n}$ ). The above equation shows that the operator $t-\mathcal{H}$ is $P$-odd, i.e. its matrix elements do not vanish only between the states of opposite parity. This makes sense since, in the static limit, $t-\mathcal{H}$ is simply $\bar{Q} i D_{z} Q$.

Using the second of the commutation relations (39) we have for the non-diagonal matrix elements of the dilatation operator $D$

$$
\left\langle k\left|t \frac{\mathrm{d}}{\mathrm{d} t}\right| n\right\rangle=-\frac{\beta^{2}}{2\left(\epsilon_{k}-\epsilon_{n}\right)^{3}} F_{0}^{(k)} F_{0}^{(n)} \cdot\left\{\begin{array}{ll}
0 & \text { wrong parity } \\
1 & \text { right parity }
\end{array} .\right.
$$


These matrix elements determine the so-called oscillator strengths - the Small Velocity (SV) transition amplitudes between the heavy quark states (usually the ground and the "P-wave" states in actual QCD). Eq. (50) allows one to prove an important symmetry relation for the IW function.

\subsection{IW function}

The IW function determines the transition amplitudes between two heavy-quark states induced by a current bilinear in two heavy quark fields. In the present context it can be defined as a diagonal scalar current in the heavy quark limit $m_{Q} \rightarrow \infty$ :

$$
\frac{1}{2 \sqrt{p_{0}^{\prime} p_{0}}}\left\langle H_{Q}^{(k)}\left(p^{\prime}\right)|\bar{Q} Q| H_{Q}^{(n)}(p)\right\rangle=\xi_{n k}\left(\frac{\left(p p^{\prime}\right)}{M_{H_{Q}}^{2}}\right), \quad \frac{\left(p p^{\prime}\right)}{M_{H_{Q}}^{2}}=\left(v v^{\prime}\right) \equiv w .
$$

In the 't Hooft model the IW functions are given by the following expression in terms of the static wavefunctions:

$$
\begin{gathered}
\xi_{n k}=\frac{2}{1+w \pm \sqrt{w^{2}-1}} \int_{0}^{\infty} \mathrm{d} t \Psi_{k}(t) \Psi_{n}\left(\left[w \mp \sqrt{w^{2}-1}\right] t\right)= \\
=\frac{2 \sqrt{z}}{1+z} \int_{0}^{\infty} \mathrm{d} t \Psi_{k}\left(\frac{t}{\sqrt{z}}\right) \Psi_{n}(\sqrt{z} t)
\end{gathered}
$$

where

$$
w=\frac{1+z^{2}}{2 z}, \quad z=w \pm \sqrt{w^{2}-1} .
$$

The expression for the IW function was obtained in Refs. [5] and [10].

Let us note that each value of $w \neq 1$ can be represented by two different values of $z$ corresponding to two possible values of the square root in Eq. (52), such that $z_{1} z_{2}=1$. They must yield the same value of $\xi$, up to a sign:

$$
\xi_{n k}(z)=P_{n} P_{k} \xi_{n k}(1 / z)
$$

which, for $n \neq k$ looks like a miraculous property of the 't Hooft equation [5]. Alternatively, the above property can be written as

$$
\xi_{n k}(w)=P_{n} P_{k} \xi_{k n}(w)
$$

Now we can demonstrate it explicitly.

Using the fact that $D \equiv t \frac{\mathrm{d}}{\mathrm{d} t}$ is the generator of scale transformations, i.e.

$$
e^{\ln (a) D} f(t) \equiv e^{\ln (a) t \frac{\mathrm{d}}{\mathrm{d} t}} f(t)=f(a t)
$$

\footnotetext{
${ }^{4}$ We thank R. Lebed for its cross checks in the numerical computations.
} 
for an arbitrary function $f(t)$, the IW function can be written in the form

$$
\xi_{n k}(z)=\frac{2 \sqrt{z}}{1+z}\left\langle k\left|\mathrm{e}^{\left(D+\frac{1}{2}\right) \ln z}\right| n\right\rangle, \quad D=t \frac{\mathrm{d}}{\mathrm{d} t} .
$$

Note that the operator $D+\frac{1}{2}$ is antihermitean (antisymmetric), so that $\| \mathrm{e}^{\left(D+\frac{1}{2}\right) \ln z}|n\rangle \|$ $=\||n\rangle \|$. This ensures the so-called first sum rule expressing the unit probability of the transition to arbitrary final state in the heavy quark limit (in the SV approximation it is known as the Bjorken sum rule).

To calculate the diagonal matrix elements of $D$ one can use the identity

$$
\int \mathrm{d} t \Psi^{\prime}(t) \Psi(t) f(t)=\frac{1}{2} \int \mathrm{d} t\left[\frac{\mathrm{d}}{\mathrm{d} t}\left(\Psi^{2}(t) f(t)\right)-\Psi^{2}(t) f^{\prime}(t)\right]=-\frac{1}{2}\left\langle f^{\prime}(t)\right\rangle
$$

valid for arbitrary $f(t)$. In particular, it shows that the expectation values of $D+\frac{1}{2}$ vanish. Together with relation (50) we see that only even powers of $D+\frac{1}{2}$ survive in the exponent in Eq. (57) when $|n\rangle$ and $|k\rangle$ have the same parity, and only odd powers contribute if the parity of the two states is opposite:

$$
\xi_{n k}=\frac{2 \sqrt{z}}{1+z} \cdot\left\{\begin{array}{ll}
\left\langle k\left|\cosh \left[\left(D+\frac{1}{2}\right) \ln z\right]\right| n\right\rangle & n-k=\text { even } \\
\left\langle k\left|\sinh \left[\left(D+\frac{1}{2}\right) \ln z\right]\right| n\right\rangle & n-k=\text { odd }
\end{array} .\right.
$$

This proves the symmetry properties Eqs. (54), (55) and ensures that the IW functions are analytic at $v v^{\prime}=1$, in spite of the branch point in $z$ as a function of $v v^{\prime}$.

\subsection{SV sum rules}

Important constraints on the transition amplitudes between heavy flavor hadrons and on the parameters of the heavy quark expansion follow from the sum rules, in particular, in the small velocity (SV) limit. In $1+1$ dimensions the first four sum rules in the heavy quark limit take the form

$$
\begin{aligned}
\rho_{k}^{2}-\frac{1}{4} & =\sum_{l} \tau_{l k}^{2} \\
\frac{1}{2} \epsilon_{k} & =\sum_{l}\left(\epsilon_{l}-\epsilon_{k}\right) \tau_{l k}^{2} \\
\left(\mu_{\pi}^{2}\right)_{k} & =\sum_{k}\left(\epsilon_{l}-\epsilon_{k}\right)^{2} \tau_{l k}^{2} \\
\left(\rho_{D}^{3}\right)_{k} & =\sum_{l}\left(\epsilon_{l}-\epsilon_{k}\right)^{3} \tau_{l k}^{2} .
\end{aligned}
$$

The so-called 'oscillator strengths' $\tau$ parameterize the transition amplitudes into the opposite-parity states in the SV limit, and $\rho^{2}$ denotes the slope of the elastic IW function:

$$
\frac{1}{2 M}\left\langle l\left|\bar{Q} \gamma_{\mu} Q\right| k\right\rangle=\tau_{l k} \varepsilon_{\mu \nu} v^{\nu}, \quad \frac{1}{2 M}\left\langle k(\vec{v})\left|\bar{Q} \gamma_{0} Q\right| k(0)\right\rangle=1-\rho_{k}^{2} \frac{\vec{v}^{2}}{2}+\mathcal{O}\left(\vec{v}^{4}\right)
$$


( $\vec{v}$ is the velocity of the final state hadron). Therefore,

$$
\tau_{l k}=\left\langle l\left|t \frac{\mathrm{d}}{\mathrm{d} t}+\frac{1}{2}\right| k\right\rangle, \quad \rho_{k}^{2}-\frac{1}{4}=\left\langle k\left|\left(t \frac{\mathrm{d}}{\mathrm{d} t}+\frac{1}{2}\right)^{2}\right| k\right\rangle .
$$

The first sum rule then becomes obvious being a consequence of completeness of the eigenstates. Other sum rules are straightforward as well.

Consider, for example the third sum rule for the kinetic operator. Using the commutator with $n=1$ in Eq. (39), we have

$$
\sum_{l} \tau_{l k}^{2}\left(\epsilon_{l}-\epsilon_{k}\right)^{2}=\sum_{l}\langle k|t-\mathcal{H}| l\rangle\langle l|t-\mathcal{H}| k\rangle=\left\langle k\left|t^{2}\right| k\right\rangle-(\langle k|t| k\rangle)^{2}=\left(\mu_{\pi}^{2}\right)_{k} .
$$

Similarly, we get for the second, "optical" sum rule

$$
\sum_{l} \tau_{l k}^{2}\left(\epsilon_{l}-\epsilon_{k}\right)=-\sum_{l}\langle k|t| l\rangle\left\langle l\left|t \frac{\mathrm{d}}{\mathrm{d} t}+\frac{1}{2}\right| k\right\rangle=-\left\langle k\left|t^{2} \frac{\mathrm{d}}{\mathrm{d} t}+\frac{t}{2}\right| k\right\rangle=\frac{\epsilon_{k}}{2} .
$$

The fourth sum rule for the Darwin operator can be directly obtained by inserting the complete set of states into the commutator $[t,[t, \mathcal{H}]]=-\frac{\beta^{2}}{2} J_{0}$ :

$$
\sum_{l} \tau_{l k}^{2}\left(\epsilon_{l}-\epsilon_{k}\right)^{3}=-\frac{1}{2}\langle k|[t,[t, \mathcal{H}]]| k\rangle=\frac{\beta^{2}\left(F^{(k)}\right)^{2}}{4}=\left(\rho_{D}^{3}\right)_{k}
$$

\section{$41 / m_{Q}$ expansion}

In practice, it is often necessary to account for the first few $1 / m_{Q}^{k}$ corrections to the static limit $m_{Q} \rightarrow \infty$, since in actuality these effects are non-negligible not only for charm, but even for beauty hadrons. In studies of the 't Hooft model there appears an additional motivation: the available numerical approaches often apply only to the finite quark masses. The solution of the static equation (23) is approximated by the solution of the generic finite-mass 't Hooft equation where $m_{Q}$ is taken large but finite. For computational reasons $m_{Q}$ cannot be taken too large, and control over the 'spurious' $1 / m_{Q}$ corrections becomes mandatory even for studies of the pure static case.

In this section we will study the leading $1 / m_{Q}$ corrections to the axial decay constant, meson masses and the kinetic energy of the heavy quark. The $1 / m_{Q}$ expansion is carried out by applying to Eq. (21) the standard formalism of non-covariant timeindependent perturbation theory used in QM. Since the leading $1 / m_{Q}$ corrections in Eq. (21) involve only powers of $t$, it is possible to derive exact expressions for the first few terms in the $1 / m_{Q}$ expansion for these observables that depend only on the moments of the static wavefunction and of the structure function. Using the results from Sect. 3, one can then express the coefficients in the $1 / m_{Q}$ expansion solely in terms of the static binding energy and the decay constant. 
Although we will perform the $1 / m_{Q}$ expansion using old-fashioned time-ordered perturbation theory, we will introduce here the corresponding notations which resemble those used in field theory, where $1 / m_{Q}$ corrections to various expectation values are given by correlators of the type

$$
-\left\langle k\left|\int_{-\infty}^{\infty} \mathrm{d} \tau i T\{A(0), \delta \mathcal{H}(\tau)\}\right| k\right\rangle
$$

Heisenberg operators $O(\tau)$ are understood as $\mathrm{e}^{i \mathcal{H} \tau} O(0) \mathrm{e}^{-i \mathcal{H} \tau}$; we assume that the Schrödinger operators we deal with, do not depend on $\tau$ explicitly.

We then denote for the stationary problem

$$
\begin{aligned}
\langle k|i T\{A, B\}| k\rangle & \equiv\left\langle k\left|\int_{-\infty}^{\infty} \mathrm{d} \tau i T\{A(0), B(\tau)\}\right| k\right\rangle \\
& =\sum_{n \neq k} \frac{\langle k|A| n\rangle\langle n|B| k\rangle}{\epsilon_{n}-\epsilon_{k}}+\sum_{n \neq k} \frac{\langle k|B| n\rangle\langle n|A| k\rangle}{\epsilon_{n}-\epsilon_{k}} .
\end{aligned}
$$

The similar expectation value can be defined for the time-ordered product of arbitrary number of operators. We consider

$$
\mathcal{H} \rightarrow \mathcal{H}+\alpha A+\beta B+\gamma C+\ldots
$$

and put

$$
\langle k|i T\{A, B, C, \ldots\}| k\rangle \equiv-\left.\left(\frac{\partial}{\partial \alpha} \frac{\partial}{\partial \beta} \frac{\partial}{\partial \gamma} \ldots \epsilon_{k}(\alpha, \beta, \gamma, \ldots)\right)\right|_{\alpha=\beta=\gamma=\ldots=0} .
$$

Two basic relations hold for such $T$-products of two operators:

$$
\langle k|i T\{[\mathcal{H}, A], B\}| k\rangle=\langle k|[A, B]| k\rangle
$$

and

$$
\begin{aligned}
\langle k|i T\{A \mathcal{H}, B\}| k\rangle & =\epsilon_{k}\langle k|i T\{A, B\}| k\rangle-\langle k|A B| k\rangle+\langle k|A| k\rangle\langle k|B| k\rangle, \\
\langle k|i T\{\mathcal{H} A, B\}| k\rangle & =\epsilon_{k}\langle k|i T\{A, B\}| k\rangle-\langle k|B A| k\rangle+\langle k|A| k\rangle\langle k|B| k\rangle .
\end{aligned}
$$

The first relation has a transparent meaning: since $[\mathcal{H}, A]=-i \frac{\mathrm{d} A}{\mathrm{~d} t}$, Eq. $(70)$ is a form of integrating by parts:

$$
\left\langle k\left|\int \mathrm{d} \tau T\left\{\frac{\mathrm{d} A(\tau)}{\mathrm{d} \tau}, B(0)\right\}\right| k\right\rangle=\left\langle k\left|\int \mathrm{d} \tau \frac{\mathrm{d}}{\mathrm{d} \tau} T\{A(\tau), B(0)\}\right| k\right\rangle+\langle k|[A(0), B(0)]| k\rangle .
$$

The obvious relation

$$
\left\langle k\left|i T\left\{\mathcal{H}^{l}, A\right\}\right| k\right\rangle=0
$$

holds as well, which will be used later. 
In the static limit, $\langle t\rangle$ equals to the bound-state energy $\epsilon$. It is often necessary to know how $\langle t\rangle$ changes under various perturbations $\delta \mathcal{H}$. The answer is readily obtained using Eqs. (39) and (70):

$$
\delta t_{k k}=\langle k|i T\{t, \delta \mathcal{H}\}| k\rangle=-\left\langle k\left|\left[t \frac{\mathrm{d}}{\mathrm{d} t}, \delta \mathcal{H}\right]\right| k\right\rangle .
$$

Since $D_{1}=t \frac{\mathrm{d}}{\mathrm{d} t}$ generates scale transformations in $t$, one finds

$$
\left[t \frac{\mathrm{d}}{\mathrm{d} t}, O\right]=\operatorname{Dim}[O] \cdot O
$$

Say, $\left[t \frac{\mathrm{d}}{\mathrm{d} t}, t^{l}\right]=l t^{l}$. Therefore, any perturbation which is a homogeneous rank- $l$ functional of $t$, satisfies 10

$$
\delta\langle t\rangle=-l\left\langle\delta \mathcal{H}_{l}\right\rangle \text {. }
$$

For example, for $\delta \mathcal{H}=\lambda t$ one finds $\delta\langle t\rangle=-\lambda$, a result that can be easily verified by direct evaluation, since the exact result reads $\langle t\rangle_{\lambda}=\langle t\rangle / \sqrt{1+2 \lambda}$.

The same property holds for non-local operators as well. For example,

$$
\langle k|i T\{t, A, B\}| k\rangle=-(D[A]+D[B]+1)\langle k|i T\{A, B\}| k\rangle,
$$

or

$$
\langle k|i T\{(t-2 \mathcal{H}), A, B\}| k\rangle=-(D[A]+D[B]-1)\langle k|i T\{A, B\}| k\rangle,
$$

where $D[A], D[B]$ denote the $t$-dimension of operators $A$ and $B$.

The above properties parallel the relations for the operator of the trace of the energy-momentum tensor for the light degrees of freedom in actual QCD discussed in Ref. [9]. This similarity was elucidated in the previous section.

The $m_{Q}$-suppressed terms in the r.h.s. of Eq. (21) playing the role of the perturbation $\delta \mathcal{H}$ are given by $t^{2} / 2 m_{Q}, t^{3} / 2 m_{Q}^{2}$, etc. Therefore, in the $1 / m_{Q}$ expansion one typically needs to compute $T$-products Eq. (68) with the operator $t^{2}$ (or $t^{3}$, in higher order). As exemplified above, this can be done directly using the relations in Eqs. (70) and (71):

$$
\begin{gathered}
\left\langle k\left|i T\left\{t^{2}, A\right\}\right| k\right\rangle=-\frac{2}{3}\left\langle k\left|\left[D_{2}, A\right]\right| k\right\rangle-\frac{4}{3} \epsilon_{k}\left\langle k\left|\left[D_{1}, A\right]\right| k\right\rangle-\frac{4}{3}\langle k|t A| k\rangle+\frac{4}{3} \epsilon_{k}\langle k|A| k\rangle, \\
\left\langle k\left|i T\left\{t^{3}-\frac{\beta^{2} J_{0}}{4}, A\right\}\right| k\right\rangle=-\frac{1}{2}\left\langle k\left|\left[D_{3}, A\right]\right| k\right\rangle-\epsilon_{k}\left\langle k\left|\left[D_{2}, A\right]\right| k\right\rangle-\frac{1}{2}\left(4 \epsilon_{k}^{2}-m^{2}+\beta^{2}\right)\left\langle k\left|\left[D_{1}, A\right]\right| k\right\rangle \\
-\frac{3}{2}\left\langle k\left|t^{2} A\right| k\right\rangle-2 \epsilon_{k}\langle k|t A| k\rangle+\frac{1}{2}\left(8 \epsilon_{k}^{2}-m^{2}+\beta^{2}\right)\langle k|A| k\rangle
\end{gathered}
$$

etc.

As an application of these relations, we obtain

$$
\left\langle k\left|i T\left\{t^{2}, t^{2}\right\}\right| k\right\rangle=-\frac{4}{3}\left(2\left\langle t^{3}\right\rangle+\langle t\rangle\left\langle t^{2}\right\rangle\right)=-\left[\frac{64}{9} \epsilon_{k}^{3}-\frac{28}{9} \epsilon_{k}\left(m^{2}-\beta^{2}\right)+\frac{2}{3} \beta^{2} F_{0}^{2}\right] .
$$


This correlator governs the $1 / m_{Q}$ corrections to the average $\left\langle t^{2}\right\rangle$ which, in turn, determines the kinetic expectation value in the static limit. Likewise

$$
\left\langle k\left|i T\left\{t^{2}, t^{l}\right\}\right| k\right\rangle=-\frac{2 l+4}{3}\left\langle k\left|t^{l+1}\right| k\right\rangle-\frac{4}{3}(l-1) \epsilon_{k}\left\langle k\left|t^{l}\right| k\right\rangle .
$$

Similarly we get the analytic expression for the $1 / m_{Q}$ corrections to the decay constants $F^{(k)}$. Indeed, $\left(F^{(k)}\right)^{2}=\left\langle k\left|J_{0}\right| k\right\rangle$, and

$$
\begin{gathered}
\delta_{1 / m_{Q}}\left(F^{(k)}\right)^{2}=\frac{1}{2 m_{Q}}\left\langle k\left|i T\left\{t^{2}, J_{0}\right\}\right| k\right\rangle= \\
\frac{1}{2 m_{Q}}\left\langle k\left|-\frac{2}{3}\left[D_{2}, J_{0}\right]\right| k\right\rangle+\frac{1}{2 m_{Q}} \frac{4}{3}\left\{\epsilon_{k}\left\langle k\left|i T\left\{t, J_{0}\right\}\right| k\right\rangle-\left\langle k\left|t J_{0}\right| k\right\rangle+\langle k|t| k\rangle\left\langle k\left|J_{0}\right| k\right\rangle\right\}= \\
\frac{1}{2 m_{Q}}\left\{-\frac{4}{3} F_{0}^{(k)} F_{1}^{(k)}-\frac{4}{3} \epsilon_{k}\left\langle k\left|\left[D_{1}, J_{0}\right]\right| k\right\rangle-\frac{4}{3} F_{0}^{(k)} F_{1}^{(k)}+\frac{4}{3} \epsilon_{k}\left(F_{0}^{(k)}\right)^{2}\right\}=-\frac{1}{2 m_{Q}} \frac{8}{3} F_{0}^{(k)} F_{1}^{(k)},
\end{gathered}
$$

where we have used that $\left[D_{1}, J_{0}\right]=J_{0}$ and $\left[D_{2}, J_{0}\right]=2 J_{1}$. Thus,

$$
\frac{\delta_{1 / m_{Q}} F^{(k)}}{F^{(k)}}=-\frac{1}{m_{Q}} \frac{2}{3} \frac{F_{1}^{(k)}}{F_{0}^{(k)}}=-\frac{2\left(2 \epsilon_{k}+m P_{k}\right)}{3 m_{Q}} .
$$

In the last equation we used relation Eq. (34) for $F_{1}^{(k)}$. The $1 / m_{Q}$ corrections to $F$ turn out significant (c.f. Refs. [5, [1]).

It is often advantageous to define the axial decay constant via the pseudoscalar current rather than the axial current:

$$
\frac{1}{2 M_{H_{Q}}}\left\langle 0\left|\bar{q} i \gamma_{5} Q\right| k\right\rangle=\frac{1}{2} \tilde{f}^{(k)}
$$

then

$$
\tilde{f}^{(k)}=f^{(k)} \frac{M_{k}}{m_{Q}+m} \quad \text { and } \quad \tilde{F}^{(k)}=F^{(k)} \frac{M_{k}}{m_{Q}+m} .
$$

Similar to what is observed in actual QCD, the $1 / m_{Q}$ corrections to the ground-state $\tilde{F}$ are smaller,

$$
\frac{\delta_{1 / m_{Q}} \tilde{F}^{(k)}}{\tilde{F}^{(k)}}=-\frac{\epsilon_{k}+m\left(3+2 P_{k}\right)}{3 m_{Q}} .
$$

The analytic expression (82) agrees with the numerical computations performed in Ref. [5] for the ground state. In terms of $c_{k}$ introduced there to quantify these preasymptotic corrections,

$$
c_{k}=\frac{5}{6} \epsilon_{k}+m P_{k}
$$

In the nonrelativistic case $\epsilon_{k} \rightarrow m$ holds, and for the negative-parity ground state one has the correct limit $c=-m / 6$ [5]. For the first excitation, however, one would have $c_{1} \rightarrow 11 \mathrm{~m} / 6$. 
It is not difficult to derive the expression for the $1 / m_{Q}$ correction to the light-cone wavefunction itself generated by perturbation $\frac{t^{2}}{2 m_{Q}}$ :

$$
\Psi_{k}(t)=\Psi_{k}^{\infty}(t)+\frac{1}{m_{Q}} \Psi_{k}^{(1)}(t)
$$

Using the commutators in Eqs. (39) we get

$$
\Psi_{k}^{(1)}(t)=\frac{1}{3}\left(t^{2} \frac{\mathrm{d}}{\mathrm{d} t}+2 \epsilon_{k} t \frac{\mathrm{d}}{\mathrm{d} t}+2 \epsilon_{k}\right) \Psi_{k}^{\infty}(t) .
$$

Note that simply replacing $m_{Q}$ by $M_{H_{Q}} \simeq m_{Q}+\epsilon$ when passing from $\varphi_{k}(x)$ to $\Psi_{k}(t)$, would amount to adding only $\frac{1}{m_{Q}} \epsilon_{k}\left(t \frac{\mathrm{d}}{\mathrm{d} t}+\frac{1}{2}\right) \Psi_{k}(t)$. The remaining part $\frac{1}{3 m_{Q}}\left(t^{2} \frac{\mathrm{d}}{\mathrm{d} t}-\epsilon_{k} t \frac{\mathrm{d}}{\mathrm{d} t}+\frac{\epsilon_{k}}{2}\right)$ actually changes the shape.

Using similar techniques, it is straightforward to obtain explicit $1 / m_{Q}$ expansion of the meson mass in the 't Hooft model; we consider it through order $\beta^{3} / m_{Q}^{2}$ corresponding to the order discussed in case of QCD [9]. A straightforward evaluation of the expectation value of Eq. (21) yields (see also [10])

$M_{H_{Q}}-m_{Q}=\langle t\rangle_{\infty}+\frac{\left\langle t^{2}\right\rangle_{\infty}-\langle t\rangle_{\infty}^{2}-\beta^{2}}{2 m_{Q}}+\frac{4\left\langle t^{3}\right\rangle+4\langle t\rangle^{3}+\left\langle i T\left\{t^{2}, t^{2}\right\}\right\rangle-4\langle t\rangle\left\langle t^{2}\right\rangle}{8 m_{Q}^{2}}+\mathcal{O}\left(\frac{\beta^{4}}{m_{Q}^{3}}\right)$.

Here all expectation values correspond to the static limit $m_{Q} \rightarrow \infty$, i.e. do not implicitly depend on $m_{Q}$. Using the previously derived relations, especially Eqs. (40) and $(79)$, all terms can be expressed only in terms of the static binding energy $\bar{\Lambda}$ and the axial decay constant of the respective state.

Finally, the object usually appearing in the $1 / m_{Q}$ expansion of the diagonal matrix elements to this order, is the zero-momentum correlator of operators $\vec{\pi}^{2}=\bar{Q}(i \vec{D})^{2} Q$ which represent the $1 / m_{Q}$ piece of the Hamiltonian,

$$
-\rho_{\pi \pi}^{3}=\frac{1}{2}\left\langle k\left|i T\left\{\vec{\pi}^{2}, \vec{\pi}^{2}\right\}\right| k\right\rangle .
$$

In particular, it determines the $1 / m_{Q}$ variation of the kinetic expectation value $\left\langle k\left|\bar{Q}(i \vec{D})^{2} Q\right| k\right\rangle$ itself in the actual finite- $m_{Q}$ hadron. The expression for $\rho_{\pi \pi}^{3}$ in the 't Hooft model is most simply obtained using the above mentioned relation $\bar{Q}\left(-i D_{z}\right) Q=t-\mathcal{H}$ which holds for the zero-momentum matrix elements in the static limit. Therefore, we simply need to compute $\left\langle k\left|i T\left\{(t-\mathcal{H})^{2},(t-\mathcal{H})^{2}\right\}\right| k\right\rangle$. In this way we obtain

$$
\begin{aligned}
-2 \rho_{\pi \pi}^{3} & =\left\langle k\left|i T\left\{\vec{\pi}^{2}, \vec{\pi}^{2}\right\}\right| k\right\rangle=4\left\langle t^{3}\right\rangle+4\langle t\rangle^{3}+\left\langle i T\left\{t^{2}, t^{2}\right\}\right\rangle-4\langle t\rangle\left\langle t^{2}\right\rangle-\frac{\beta^{2}}{2} F^{2}= \\
& =-\left(\frac{4}{9} \epsilon_{k}^{3}-\frac{4}{9} \epsilon_{k}\left(m^{2}-\beta^{2}\right)+\frac{1}{6} \beta^{2} F^{2}\right)=-\left(\frac{4}{3} \mu_{\pi}^{2} \epsilon_{k}+\frac{1}{6} \beta^{2} F^{2}\right) .
\end{aligned}
$$


This correlator is numerically large.

We can compare the $1 / m_{Q}$ expansions discussed above with the general operator expansion Ref. [9, 7] valid in arbitrary gauge theory:

$$
M_{H_{Q}}-m_{Q}=\bar{\Lambda}+\frac{\mu_{\pi}^{2}-\beta^{2}}{2 m_{Q}}-\frac{1}{m_{Q}^{2}}\left[-\frac{1}{8}\left\langle i T\left\{\vec{\pi}^{2}, \vec{\pi}^{2}\right\}\right\rangle+\frac{\rho_{D}^{3}}{4}\right]+\ldots, \quad \rho_{D}^{3}=\frac{\beta^{2} F^{2}}{4} .
$$

Similarly - and even a bit simpler - is to consider the expansion of the scalar density which is given precisely by [7]

$$
\frac{1}{2 M_{H_{Q}}}\langle\bar{Q} Q\rangle=\frac{m_{Q}}{M_{H_{Q}}}\left\langle\frac{1}{x}\right\rangle
$$

and, on the other hand, use the similar OPE for $\langle\bar{Q} Q\rangle$ :

$$
\frac{1}{2 M_{H_{Q}}}\left\langle H_{Q}|\bar{Q} Q| H_{Q}\right\rangle=1-\frac{\left\langle\bar{Q}(i \vec{D})^{2} Q\right\rangle-\beta^{2}}{2 m_{Q}^{2}}-\frac{\rho_{D}^{3}}{2 m_{Q}^{3}}+\mathcal{O}\left(\frac{\beta^{4}}{m_{Q}^{3}}\right) .
$$

The explicit computations show that these equations are satisfied with $\rho_{\pi \pi}^{3}$ given by Eq. (91).

It is interesting that it is possible to derive a closed expression for the expectation value of the 'kinetic' operator $\bar{Q}\left(i D_{z}\right)^{2} Q$ in terms of the 't Hooft wavefunction for arbitrary mass $m_{Q}$ :

$$
\begin{gathered}
\frac{1}{2 M_{H_{Q}}}\left\langle H_{Q}\left|\bar{Q}\left(i D_{z}\right)^{2} Q\right| H_{Q}\right\rangle= \\
\frac{m_{Q}}{2 M_{H_{Q}}}\left[M_{H_{Q}}^{2}\langle x\rangle-m_{Q}^{2}\left\langle\frac{1}{x}\right\rangle+\frac{\beta^{2}}{4}\left(\frac{M_{H_{Q}}^{2}}{m_{Q}\left(m_{Q}-m P\right)} \int_{0}^{1} \mathrm{~d} x \varphi(x)\right)^{2}\right]
\end{gathered}
$$

(this expression assumes a certain ultraviolet regularization of the operator, see below), where $P$ is parity of $H_{Q}$. The idea is the following.

In the rest frame of the meson the expectation value of $\bar{Q}\left[\left(i D_{0}\right)^{2}+\left(i D_{z}\right)^{2}\right] Q$ is simply $2\left\langle\bar{Q}\left(i D_{-}\right)^{2} Q\right\rangle$ and, therefore

$$
\frac{1}{2 M_{H_{Q}}}\left\langle H_{Q}\left|\bar{Q}\left[\left(i D_{0}\right)^{2}+\left(i D_{z}\right)^{2}\right] Q\right| H_{Q}\right\rangle=m_{Q} M_{H_{Q}} \int_{0}^{1} \mathrm{~d} x x \varphi^{2}(x) .
$$

The complementary combination of momentum operators $\bar{Q}\left[\left(i D_{0}\right)^{2}-\left(i D_{z}\right)^{2}\right] Q$ can be determined using the general identity

$$
\int d^{D} x \bar{Q} Q(x)=\int d^{D} x\left\{\bar{Q} \gamma_{0} Q+\bar{Q} \frac{\left(i D_{0}-m_{Q}\right)^{2}-(i \vec{D})^{2}+\frac{i}{2} \sigma^{\mu \nu} G_{\mu \nu}}{2 m_{Q}^{2}} Q\right\}
$$

valid in arbitrary dimension. In $D=2$ one has $\frac{i}{2} \sigma^{\mu \nu} G_{\mu \nu}=\tilde{G} i \gamma^{5}$ with $\tilde{G}=\frac{1}{2} \epsilon^{\mu \nu} G_{\mu \nu}=$ $G_{01}$. Since $\left\langle\bar{Q} i D_{z} Q\right\rangle=\left\langle\bar{Q} i D_{0} i D_{z} Q\right\rangle=0$ and the light-cone combination $\left\langle\bar{Q}\left(i D_{0}-\right.\right.$ 
$\left.\left.i D_{z}\right) Q\right\rangle$ is again directly expressed via the 't Hooft wavefunction, Eq. (97) yields the necessary equation for $\left\langle\bar{Q}\left[\left(i D_{0}\right)^{2}-\left(i D_{z}\right)^{2}\right] Q\right\rangle$ involving, however the expectation value $\left\langle\bar{Q} \tilde{G} i \gamma^{5} Q\right\rangle$.

The gluonic field strength $G_{01}$ can be explicitly written via quark current in the light-cone gauge:

$$
G_{01}=-g_{s}^{2} \frac{1}{\partial_{-}} J^{+}
$$

which leads to a non-local four-fermion operator. We note, however, that the current $J^{+}$includes not only the spectator quark $q$, but also $\bar{Q} \gamma^{+} \frac{\lambda^{a}}{2} Q$. This term described by the bare loop leads to the ultraviolet divergent expression. We simply discard this contribution in $J^{+}$in Eq. (98), which fixes a certain renormalization procedure. Then we get

$$
\begin{aligned}
\left\langle\bar{Q} \frac{i}{2} \sigma^{\mu \nu} G_{\mu \nu} Q\right\rangle=\left\langle\bar{Q} \tilde{G} i \gamma^{5} Q\right\rangle & =\frac{\beta^{2}}{4} \frac{m_{Q}}{M_{H_{Q}}} \int_{0}^{1} \mathrm{~d} x \varphi(x) \int_{0}^{1} \mathrm{~d} y \varphi(y) \frac{1}{x-y}\left(\frac{1}{x}-\frac{1}{y}\right) \\
& =-\frac{\beta^{2}}{4} \frac{m_{Q}}{M_{H_{Q}}}\left(\int_{0}^{1} \frac{\mathrm{d} x}{x} \varphi(x)\right)^{2} .
\end{aligned}
$$

This finally yields Eq. (95) where we have used Eqs. (14) to express the last term via the decay constant of the meson.

A note of caution must be voiced regarding this derivation, however. In the way described above we obtain the bare operator $\bar{Q}\left(i D_{z}\right)^{2} Q$. It does include a finite contribution from the domain of momenta of order $m_{Q}$ even in the leading order in $m_{Q}$. On the contrary, in the heavy quark expansion we are interested only in the physics originating from momenta essentially below the scale of the heavy quark mass itself. The expressions Eqs. (90), (91) refer just to such low-energy effective operator. Therefore, in general the literal comparison of the two expectation values is not too instructive. It is easy to check that already to the leading order in $m_{Q}$ the two expressions differ by the amount $\frac{\beta^{2}}{2}$ attributed to the domain of momenta $\sim m_{Q}$.

Here we note an interesting feature of the exact expectation value of the local quark-gluon operator $\bar{Q} \tilde{G} i \gamma_{5} Q$ in Eq. (99). At $m_{Q} \rightarrow 0$ it has an $1 / m_{Q}$ singularity regardless of the mass of the second quark in the meson: $\int_{0}^{1} \mathrm{~d} x / x \varphi(x) \propto 1 / m_{Q}$ at $m_{Q} \ll \beta$. Yet we know that no appropriate massless physical states exist in the model at $m_{q} \neq 0$ (the ground $Q \bar{Q}$ state has negative parity, and their pairs are $1 / N_{c}$ suppressed). The singularity technically emerges due to massless gluon propagator, however gluon is absent from the physical spectrum in $D=2.0$

It must be noted, however, that careful treatment of passing to the light-cone coordinates in the computations of the similar vacuum expectation value $\left\langle 0\left|\bar{\psi} \tilde{G} i \gamma_{5} \psi\right| 0\right\rangle$ in the 't Hooft model yielded additional terms which canceled the $1 / m_{\psi}$ pole observed

\footnotetext{
${ }^{5}$ We are grateful to A. Vainshtein for informing us of existing examples of similar IR singularities in physical amplitudes in the absence of contributing massless particles, in low dimensions. Reportedly, such a situation is excluded in $D>3$.
} 
in Ref. 12 and led to a finite result at $m_{\psi} \rightarrow 0$. A possibility of similar subtleties in the computation of the meson expectation values deserves further studies. We are grateful to A. Zhitnitsky for pointing out and discussing this problem.

\section{1 $1 / m_{Q}^{2}$ correction at zero recoil}

As another application of the $1 / m_{Q}$ expansion, we briefly consider here the secondorder nonperturbative corrections to the zero-recoil $B \rightarrow D^{(*)}$ transition amplitude. At this kinematic point the deviation from the elastic IW function (which is unity here) appears at the level $1 / m_{c, b}^{2}$, which provides a method of extracting $\left|V_{c b}\right|$. The corrections, however, are shaped by strong dynamics at the typical hadronic scale and at present cannot be evaluated from the first principles. The existing estimates, in particular for the axial $B \rightarrow D^{*}$ amplitude, rely on the sum rules derived in Refs. [9, 13]:

$$
\left|F_{D^{*}}\right|^{2}+\sum_{k \neq 0}\left|F_{k}\right|^{2}=\xi_{A}-\frac{\mu_{G}^{2}}{3 m_{c}^{2}}-\frac{\mu_{\pi}^{2}-\mu_{G}^{2}}{4}\left(\frac{1}{m_{c}^{2}}+\frac{1}{m_{b}^{2}}+\frac{2}{3 m_{c} m_{b}}\right)+\mathcal{O}\left(\frac{1}{m_{Q}^{3}}\right)
$$

where $F_{k}$ are the zero-recoil transition amplitudes to the excited states, $F_{k} \propto 1 / m_{Q}$, $\xi_{A}$ is the short-distance renormalization factor, and $\mu_{\pi}^{2}, \mu_{G}^{2}$ are expectation values of the kinetic and chromomagnetic operators, respectively. One then has [13]

$$
F_{D^{*}}=\xi_{A}^{1 / 2}-\left[\frac{\mu_{G}^{2}}{6 m_{c}^{2}}+\frac{\mu_{\pi}^{2}-\mu_{G}^{2}}{8}\left(\frac{1}{m_{c}^{2}}+\frac{1}{m_{b}^{2}}+\frac{2}{3 m_{c} m_{b}}\right)\right] \cdot(1+\chi)+\mathcal{O}\left(\frac{1}{m_{Q}^{3}}\right),
$$

where a positive quantity $\chi$ parameterizes the magnitude of the sum of the excitation

probabilities in the 1.h.s. of the sum rules, in terms of the local operator term in the r.h.s. of Eq. (100):

$$
\sum_{k \neq 0}\left|F_{k}\right|^{2}=\chi \cdot\left[\frac{\mu_{G}^{2}}{3 m_{c}^{2}}+\frac{\mu_{\pi}^{2}-\mu_{G}^{2}}{4}\left(\frac{1}{m_{c}^{2}}+\frac{1}{m_{b}^{2}}+\frac{2}{3 m_{c} m_{b}}\right)\right] .
$$

The expressions for the excitation amplitudes were elaborated in Ref. [9]. Following Ref. [13], existing numerical estimates of $F_{D^{*}}$ assume (somewhat arbitrarily) that $\chi$ can vary up to 1 , that is, $\chi=0.5 \pm 0.5$.

We computed $\chi$ analytically in the 't Hooft model. Since spin and chromomagnetic field are absent in two dimensions, only the kinetic operator acts here. The sum rule takes the form

$$
F_{D}^{2}+\sum_{k \neq 0}\left|F_{k}\right|^{2}=\xi_{A}-\left(\frac{1}{m_{c}}-\frac{1}{m_{b}}\right)^{2} \frac{\mu_{\pi}^{2}}{4}+\mathcal{O}\left(\frac{1}{m_{Q}^{3}}\right)
$$

and the excitation amplitudes $F_{k}$ to the leading order are given by

$$
F_{k}=\frac{1}{2}\left(\frac{1}{m_{c}}-\frac{1}{m_{b}}\right) \frac{\left\langle k\left|\bar{Q} \pi_{z}^{2} Q\right| 0\right\rangle}{\epsilon_{k}-\epsilon_{0}}
$$


Similarly, $\chi$ is defined through

$$
\sum_{k \neq 0}\left|F_{k}\right|^{2}=\chi \cdot\left(\frac{1}{m_{c}}-\frac{1}{m_{b}}\right)^{2} \frac{\mu_{\pi}^{2}}{4} .
$$

The sum of $F_{k}^{2}$ can be computed using the same technique as was elaborated in the previous sections. However, the corrections to the amplitudes we consider are not expressed anymore via only positive integer moments, and include expectation values of operators with higher derivatives. Yet they can be expressed in terms of the slope of the IW function $\rho^{2}$. We give here the final result

$$
\chi=\frac{10}{21}+\frac{5}{63} \frac{\epsilon_{0}^{2}}{\mu_{\pi}^{2}}-\frac{4}{21}\left(\rho^{2}-\frac{1}{4}\right)=\frac{5}{7}+\frac{5}{21} \frac{m^{2}-\beta^{2}}{\epsilon_{0}^{2}-m^{2}+\beta^{2}}-\frac{4}{21}\left(\rho^{2}-\frac{1}{4}\right) .
$$

For light spectator quark the value of $\chi$ turns out to be about 0.55 . This is surprisingly close to the central value guestimated in Ref. [13] for the case of actual QCD.

\section{Summary and Outlook}

We have studied the 't Hooft model in the $m_{Q} \rightarrow \infty$ limit. Our main result are exact relations for the heavy quark kinetic energy, as well as moments of the 't Hooft wavefunction and of the structure function in this limit, which allow to express these observables in terms of only the heavy quark binding energy $\bar{\Lambda}$ and the axial decay constant $F^{k}$.

In the 't Hooft model, these moments appear as coefficients in the $1 / m_{Q}$ expansion for various observables. As an example, we calculated coefficients in the $1 / m_{Q}$ expansion of meson masses, decay constants and heavy quark kinetic energies. Using the above relations, we were able to express the corresponding $1 / m_{Q}$ coefficients in terms of $\bar{\Lambda}$ and $F^{k}$.

Likewise, we derived the expressions for the oscillator strengths and verified a set of the SV sum rules in the heavy quark limit. As an application of the developed $1 / m_{Q}$ expansion, we computed the nonperturbative $1 / m_{Q}^{2}$ corrections to the zero recoil $B \rightarrow D$ transition amplitude.

Although the 't Hooft model is in principle "numerically solvable", many observables can only be determined with very limited precision in practical calculations. This is particularly the case for observables in the limit where one of the quarks becomes heavy. In this case the 't Hooft wavefunction becomes extremely asymmetric and many numerical techniques, which are otherwise rather powerful for finite

quark masses, fail to produce numerically reliable results. For this regime, where the heavy quarks are not infinitely heavy, it is often advantageous to perform the $1 / m_{Q}$ expansion beyond the leading order. The corresponding expansion coefficients that we derived involve only properties of the 't Hooft wavefunctions in the static limit. 
Moreover, through the use of exact relations, the expansion coefficients can be expressed in terms of $m_{Q} \rightarrow \infty$ properties of the wavefunctions that can be calculated numerically with sufficiently high accuracy.

The developed analytic $1 / m_{Q}$ expansion allows to carry out precision studies, in the framework of the 't Hooft model, of such an intriguing and poorly understood phenomenon as violation of local duality in heavy flavor decays [14]. The question of its magnitude has a particular phenomenological significance in the domain of moderately heavy quarks, where reliability of the asymptotic expansions is unknown a priori, and numerical computations are unavoidable.

The developed technique can be used to test, on the example of the 't Hooft model, various approximations routinely applied to the actual beauty decays. Some of them will be reported in Ref. [14], while others further deserve dedicated studies.

Acknowledgments: This work has been supported by the NSF under grant number PHY96-05080, by the DOE under grant number DE-FG03-95ER40965, by NATO under the reference PST.CLG 974745, by RFFI grant \# 99-02-18355, and in part by TJNAF. N.U. thanks I. Bigi, R. Lebed, M. Voloshin and A. Zhitnitsky for helpful comments and M. Shifman and A. Vainshtein for encouraging interest. N.U. gratefully acknowledges the hospitality of Physics Department of the Technion and the support of the Lady Davis grant during completion of this paper.

\section{References}

[1] A concise review of the heavy quark expansion is given in: I.I. Bigi, M. Shifman and N. Uraltsev, Annu. Rev. Nucl. Part. Sci. 47 (1997) 591.

[2] G. 't Hooft, Nucl. Phys. B75 (1974) 461.

[3] C. Callan, N. Coote and D. Gross, Phys. Rev. D13 (1976) 1649.

[4] M. Burkardt, Nucl. Phys. A 504762 (1989); M. Burkardt, Ph. D. thesis, Univ. Erlangen, 1989.

[5] M. Burkardt and E. Swanson, Phys. Rev. D46 (1992) 5083.

[6] M. Burkardt, Phys. Rev. D46 (1992) R1924 and R2751.

[7] I. Bigi, M.Shifman, N. Uraltsev and A. Vainshtein, Phys. Rev. D59 (1999) 054011.

[8] N. Uraltsev, Phys. Lett. B376 (1996) 303.

[9] I. Bigi, M. Shifman, N. Uraltsev and A. Vainshtein, Phys. Rev. D52 (1995) 196.

[10] I. Bigi and N. Uraltsev, Phys. Rev. D 60 (1999) 114034. 
[11] B. Grinstein and P.F. Mende, Phys. Rev. Lett. 69 (1992) 1018.

[12] B. Chibisov and A. Zhitnitsky, Phys. Lett. B362 (1995) 105.

[13] M. Shifman, N.G. Uraltsev and A. Vainshtein, Phys. Rev. D51 (1995) 2217.

[14] R. Lebed and N. Uraltsev, in preparation. 